\title{
Fuzzy Algorithm for Human Drowsiness Detection Devices
}

\author{
Simona Dzitac, Tiberiu Vesselenyi, Laurentiu Popper, Ioan Moga, Calin Dinu Secui \\ Faculty of Energy Engineering, University of Oradea, \\ 1 Universității Street, Oradea, Romania \\ simona.dzitac@gmail.com
}

\begin{abstract}
There are several human activities where the awareness and conscious control is a very important factor: vehicle driving, heavy equipment operation, hazardous materials manipulation. In these cases drowsiness can be the cause of injury or even death. For example car driver drowsiness is one of the causes of serious traffic accidents, which makes this an area of a significant importance. Continuous monitoring of driver's or operator's drowsiness is of great importance if we want to reduce accidents due to operator's fault. If drowsiness is detected in time, a significant part of these accidents could be successfully prevented. In the last years various methods were tested, based on the use of: heart rate variability, video monitoring of the eyes, EEG, EMG and ECG signals.
\end{abstract}

Our research is based on the study of EEG and EMG signals and aims to develop algorithms capable to detect features specific to the drowsiness state and decide the moment in which the driver or operator should be alerted.

Keywords: drowsiness alert, EEG, EMG, fuzzy decision algorithm.

\section{Introduction}

As it was reported in several studies $60 \%$ of adult drivers have driven while felling drowsy in the past years, and $37 \%$ have actually fallen asleep while driving. For this reason, a technique that can detect the driver's drowsiness in real-time is very important in order to prevent accidents caused by drowsiness. In cases of equipment operation there are no statistics published but the danger of accidents is also present. If drowsiness could be detected, incidents can be prevented by countermeasures in order to awake the driver or the operator.

In order to analyze the state of awareness it is important to know the phases of sleep. Sleep cycle is divided into Non-Rapid Eye Movement (NREM) sleep and Rapid Eye Movement (REM) sleep, and the NREM sleep is further divided into four stages. Drowsiness is the first stage of sleep in the NREM domain $[1,2]$. So the detection of awareness can be based, among others, on the distinction between first stage of NREM state and awake state.

In the last years a large number of methods had been tested, based on the use of: heart rate variability, video monitoring of the eye movement and facial expression, EEG, EMG and ECG signals.

The video monitoring approach analyzes the images captured by cameras to detect physical changes of driver's image, such as eyelid movement, eye gaze, and head nodding. Some systems are using cameras and imaging processing techniques to measure the percentage of eyelid closure over time. Although this vision based method is not invasive and is not causing annoyance to drivers, the drowsiness detection accuracy is severely affected by the environmental backgrounds, driving or operating conditions and also requires the camera to focus on a relatively small area.

The physiological signal detection approach is to measure the changes of driver or operator biological signals, such as the electroencephalography (EEG) and electrocardiogram (ECG). Since the sleep rhythm is strongly correlated with brain and heart activities (brain rhythms correlated with NREM first stage), these physiological signals can give more accurate drowsiness detection than video monitoring. The drawback of this method is that the electrode contacts and wires can make a discomfort for the driver or operator and also that the electrodes must be placed on the skull using a conductive gel which ensures a good contact with the skin. These difficulties can be overcome by using dry-contact, low-noise EEG sensors, as it is described in [3]. The dry-contact EEG electrodes were created by the authors [3] with micro-electricalmechanical system (MEMS) technology. Each channel of the analog signal processing front-end comes on a custom-built, smallsized circuit board which contains an 
amplifier, filters, and analog-to-digital conversion. As the authors describe daisychain configuration between boards with bitserial output reduces the wiring needed and the system is capable to detect alpha-band rhythms and eye-blink signals.

Also for driver awareness detection a number of methods were proposed which are based on the technique of embedding biosensors into steering wheel or in the seat of the vehicle in order to measure heart beat pulse signals [4]. Time series of heart beat pulse signal can be used to calculate the heart rate variability. As it is shown in [4], the frequency domain spectral analysis of heart rate variability shows that typical it has three main frequency bands: high frequency band, low frequency band and very low frequency. A number of psycho-physiological researches have found that the low and high frequency power spectral density ratio decreases when a person changes from waking into drowsiness/sleep stage, while the high frequency power increases associated with this status change. The authors in [4] show that this variability can be an effective method for the detection of driver drowsiness. The problem with this method is that the drowsiness detection equipment is not portable and it must be embedded in the vehicle parts which will limit its applicability. is used, and can be applied both for drivers and equipment operators too.

\section{Experimental Setup and Method}

Subjects had to fulfil two different tasks. The first one is related to eye blinking and eye movement and the second task is related to reaching a highly relaxed state close to drowsiness, while monitoring the physiological signals.

For the first task the subject had to blink the eyes in a specific rhythm $(f 1)$ for a specified number of times $(n 1)$ and then change the rhythm $(f 2)$ for another number of times $(n 2)$. An ideal diagram of eye-blink rhythms can be seen in Figure 1. A similar paradigm has been used for eye movement experiments.

For example in Figure 1:

$$
f_{1}=1 / \Delta t_{1} ; f_{2}=1 / \Delta t_{2} ; n_{1}=4 ; n_{2}=2 \text {. }
$$

The second task is that of producing an EEG signal with a major alpha rhythm component by reaching a highly relaxed state. This had been done by requesting the subject to produce an alert (aware) state (for example being attentive to images recorded during a vehicle running on a road) and then in a different measurement session a totally relaxed state (eves closed and emntv minded).

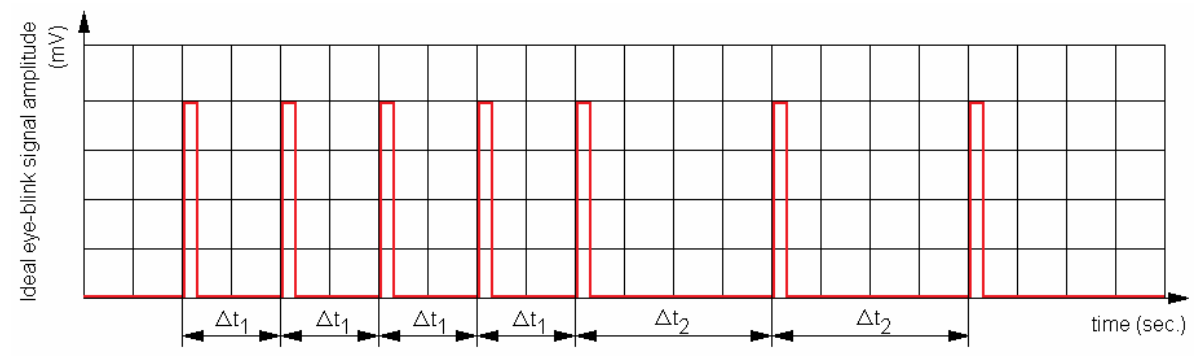

Figure 1. Expected eye blink signal.

Analyzing the above mentioned approaches we decided to study algorithms which can be used for drowsiness/awareness detection when applying techniques based on EEG and EMG signals measurement. There are also a large number of studies which uses EEG signals for brain - computer interface applications $[5,6,7,8]$. If the EEG device is built in the manner described in [3] this method has the advantage that is independent from the equipment, vehicle or situ where it
A usual diagram for alert state is shown in Figure 2, and a diagram of relaxed state is shown in Figure 3. 


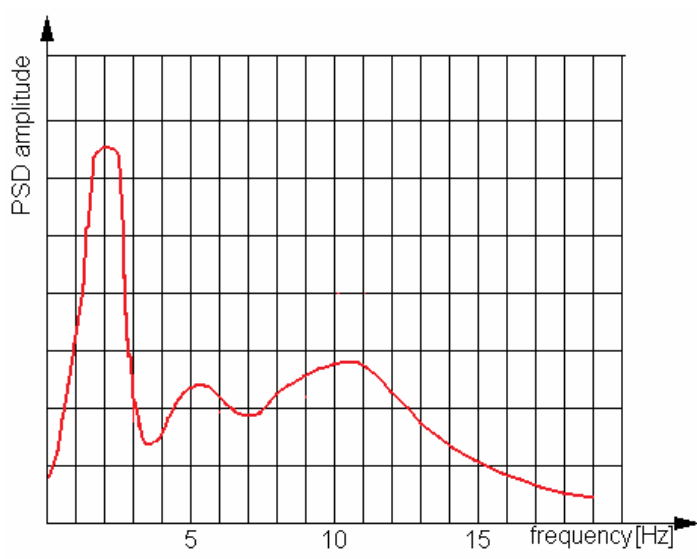

Figure 2. Expected shape of power spectral density (PSD) in aware state.

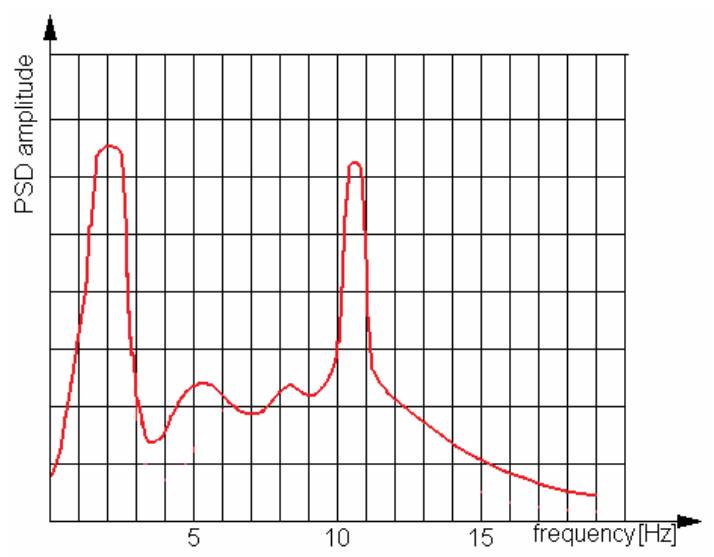

Figure 3. Expected shape of power spectral density (PSD) in relaxed state.

A total of five healthy male adults (aged 2548) participated in the study. None of the subjects had participated in such experiments before and the subjects had a short basic training for the task. The subjects were informed about the nature and purpose of the experiments and consented to participate.

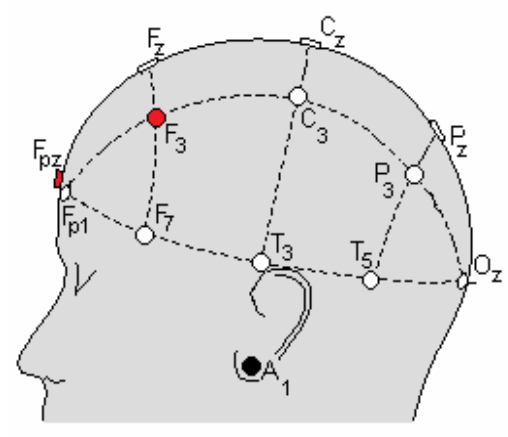

PC, a National Instruments NI-USB 6251 DAQ board has bean used. The accuracy of the DAQ board is of $15.259 \mu \mathrm{V}$. The Ag$\mathrm{AgCl}$ electrodes were placed in the $\mathrm{Fp} 1$ point for the eye blinking and eye movement detection and in the F3 point on the skull for alpha rhythm measurement, using a conductive gel (Figure 4). As voltage reference, the A1 point was used (Figure 4) and the ground was located on the right leg. Data were sampled at a sample rate of 1000 $\mathrm{Hz}$ and were transmitted through the USB port to a PC for storage and processing. The data acquisition and processing programs were written in MATLAB language.

Every subject has made 3 training sessions to accommodate with the environment and the equipment and then 4 sessions which were recorded.

\section{Experimental Results}

The useful potentials measured on the skull are considerably low. In case of EMG (when measuring eye movement or blinking) is about from hundreds to a few thousands of micro volts. EEG potentials are much lower from a few to a hundred micro volts. The signal conditioning methods and equipments (amplification and filtering) has to be of a very good quality at these low levels of potential. Even so due to the electrical complexity of the human body, noises from different sources can alter the useful signals. One of the major noises which are present in these signals (but may be avoided if the system described in [3] is used) is the $50 \mathrm{~Hz}$

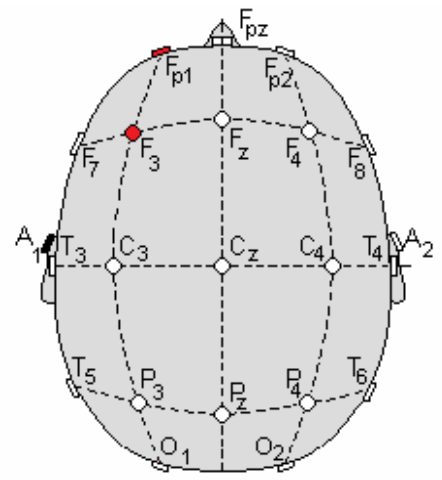

Figure 4. Electrode placement used in the experiments (pointed out by the red dots).

EEG signals were acquired using a BST112 8-channel amplifier from VEB MESSGERATEWERK ZVONIZ and for $\mathrm{A} / \mathrm{D}$ conversion and transmission of data to a component which is due to the AC source of the EEG device. These noises are observable in Figure 5 and Figure 8 time diagrams. In Figure 8 the noise is much larger compared to 
the useful signal, because the eye movement signals are much lower than the eye blink signals. Another components which can be considered as noises are the heart beat (or pulse) signals which can not be always avoided. An example of such signals can be seen in Figure 10. Figures 6 and 9 show the Power Spectral Density of signals in Figures 5 and 8. Studying the possibilities of filtering these signals we can assume that filtering eye blink and eye movement can be made using frequency domain filtering using the same filtering parameters both for eye blinking and eye movement signals.

Regarding the EEG signals these were recorded both in aware and drowsy states as described in section 2. An example of aware state signal is shown in Figure 12 and an example of drowsy state signal is given in Figure 14. From these signals we can obtain the PSD using a simple FFT algorithm. The PSD Amplitude versus frequency diagrams are shown in Figures 13 and 15. Analyzing these diagrams we can observe (as it is also shown by different authors) that the peak representing the alpha rhythm (in the frequency domain of 8-12 $\mathrm{Hz}$ ) clearly increases (almost doubles) in drowsy state compared to aware state.

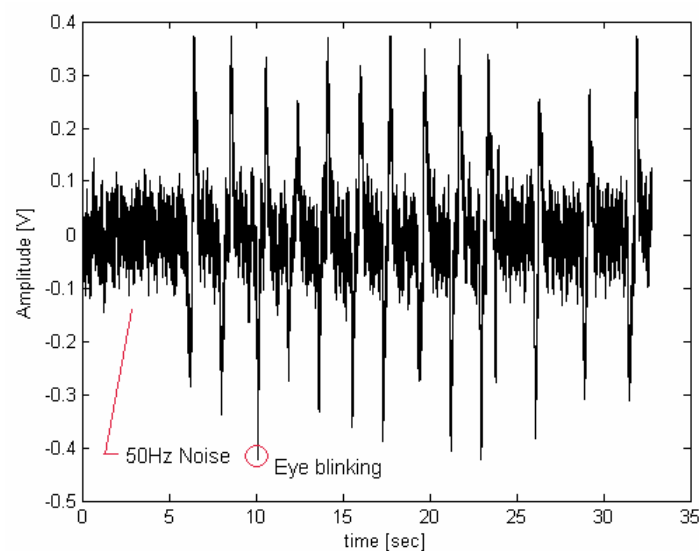

Figure 5. Eye blink time signal, showing 10 eye blinks at intervals of 1.5 seconds and 3 eye blinks at intervals of 2.5 seconds.

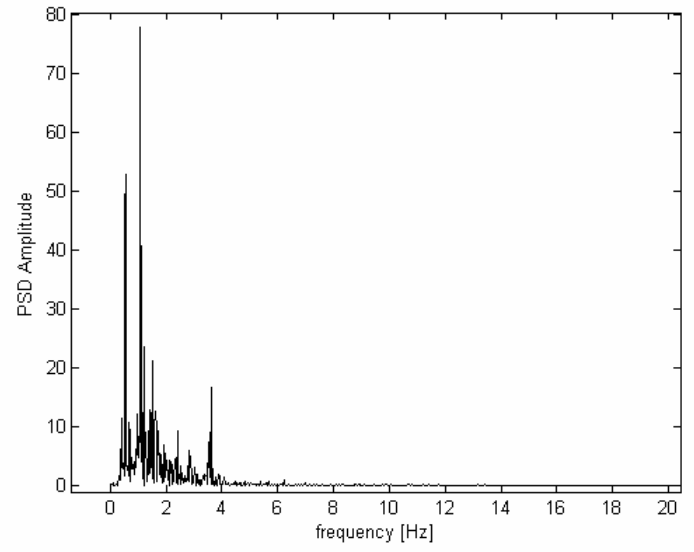

Figure 6. Eye blink PSD amplitude versus frequency of the signal presented in Figure 5.

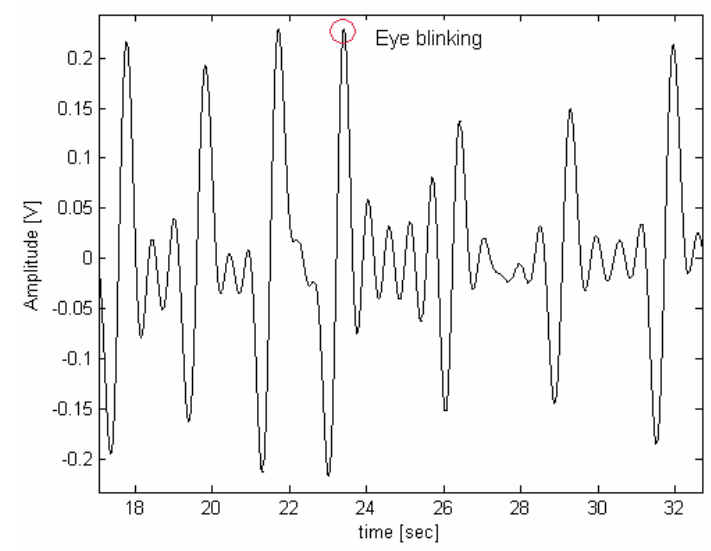

Figure 7. Eye blink time signal from Figure 5, filtered with a low pass FFT filter in the 0-6 Hz domain.

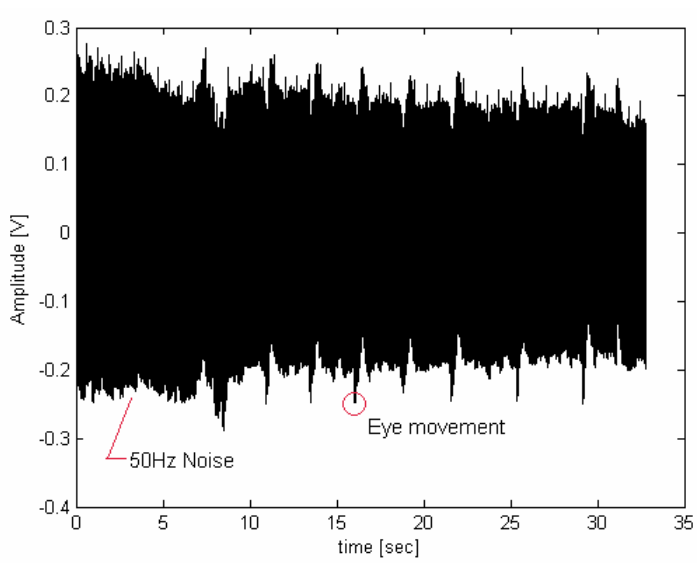

Figure 8. Eye movement time signal, showing 6 eye movements at intervals of 2 seconds and 2 eye movements at intervals of 3 seconds. 


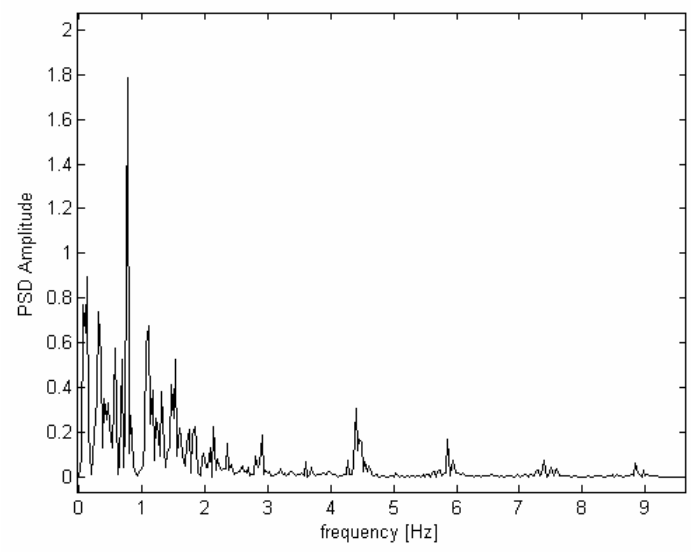

Figure 9. Eye movement PSD amplitude versus frequency of the signal presented in Figure 8.

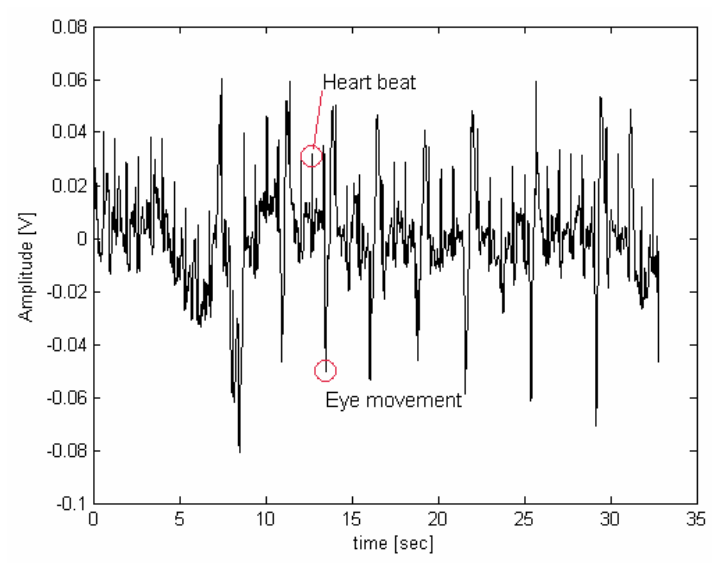

Figure 10. Eye movement and heart beat time signal from Figure 8, filtered with a low pass FFT filter in the $0-20 \mathrm{~Hz}$ domain.

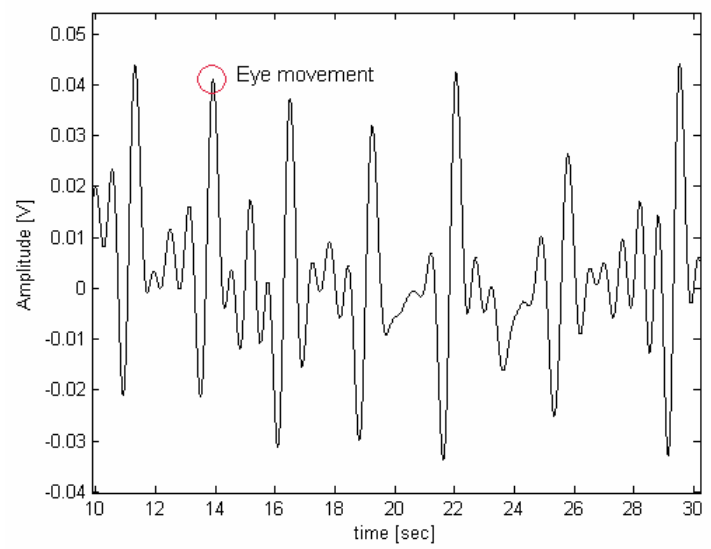

Figure 11. Eye movement time signal from Figure 8, filtered with a low pass FFT filter in the 0-6 Hz domain.

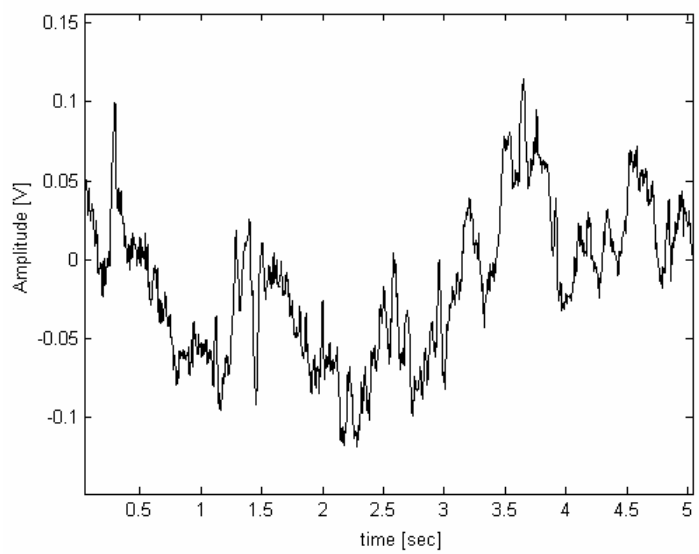

Figure 12. EEG signals recorded as described in section 2. Aware state.

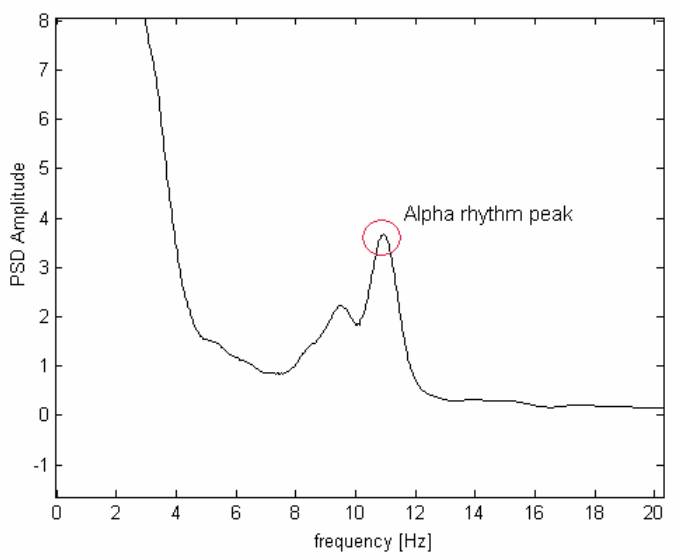

Figure 13. PSD amplitude versus frequency of the EEG signal presented in Figure 12, with the alpha rhythm peak in the $10-12 \mathrm{~Hz}$ frequency domain.

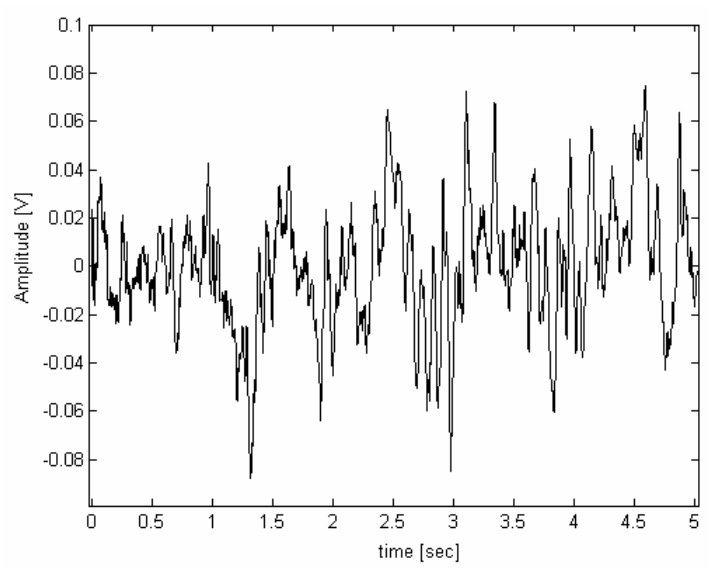

Figure 14. EEG signals recorded as described in section 2. Drowsy state. 


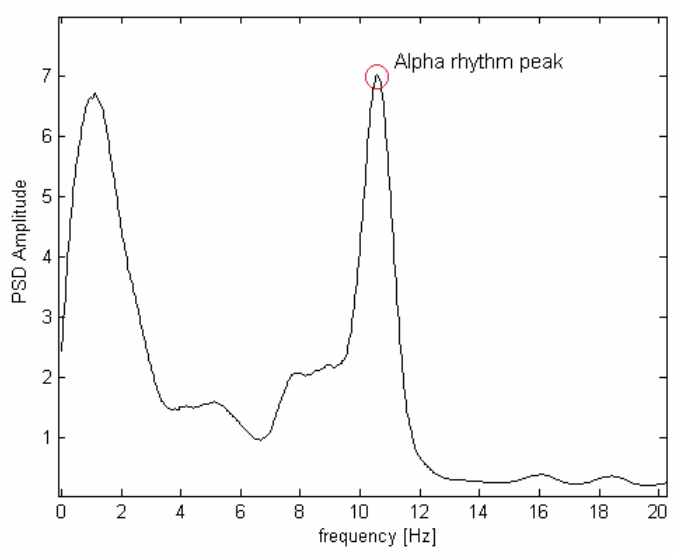

Figure 15. PSD amplitude versus frequency of the EEG signal presented in Figure 14, with the alpha rhythm peak in the $10-12 \mathrm{~Hz}$ frequency domain.

\section{Data Processing and Fuzzy Decision Algorithm.}

Our approach is based on the observation that the condition of drowsiness is on one hand the first stage of the NREM stage that involve the absence of any eye movement or eye blinking signal and on the other hand, the increasing PSD amplitude in the alpha rhythm $(8-12 \mathrm{~Hz})$ frequency band. We consider that these two features are sufficient to discriminate between the awareness and drowsiness conditions.

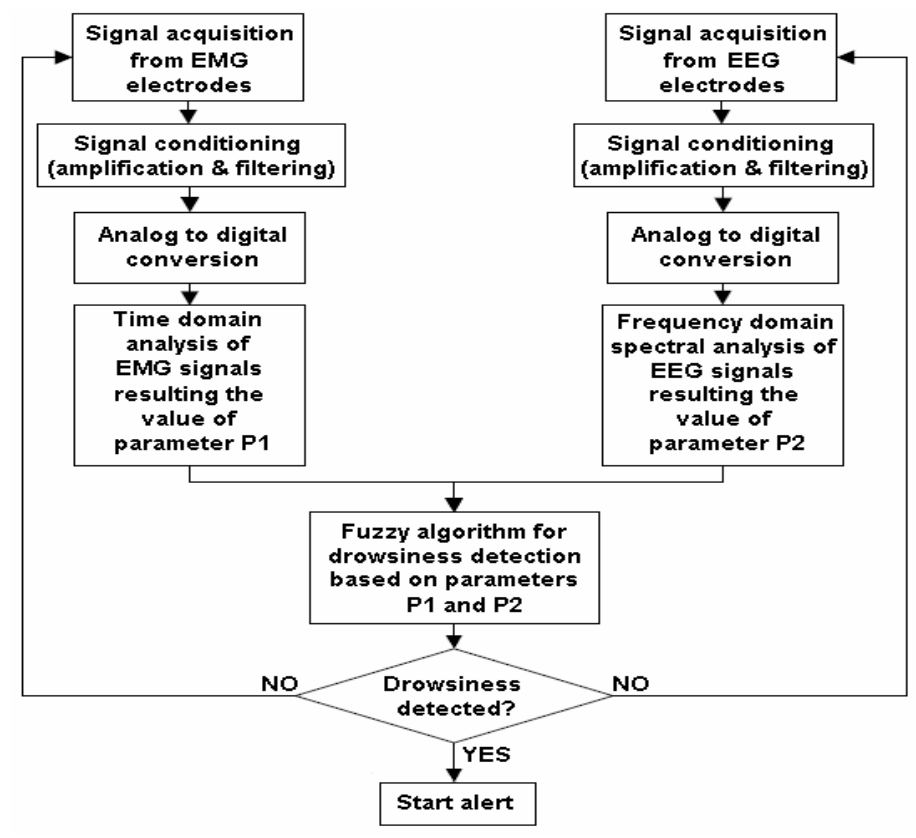

Figure 16. Flow chart of the drowsiness detection algorithm.
The drowsiness detection system which we propose is presented in Figure 16. The eye blink and eye movement is detected by the same electrode and the acquisitioned signals are filtered in the same way for both blinking and movement, as described in section 3 .

Once the eye blink and movement are obtained in the form shown in Figures 7 and 11, these can be analyzed using a detection algorithm which computes the current value of the decision parameter P1 using the expression:

$$
P 1=\frac{A(s)}{R M S(i)}
$$

where:

- $A(s)$ - is the time signal amplitude at time $\mathrm{s}$;

- RMS(i) - is the root mean square of the time signal amplitude for the domain $i \in[s-n, s]$;

The EEG signals are processed using the FFT algorithm and then the $\mathrm{P} 2$ parameter value is computed with the expression:

$$
P 2=\frac{\sum_{j}^{j+m} \operatorname{Re}(F F T(n))}{m}
$$

where: $\mathrm{j}=8 \mathrm{~Hz} ; \mathrm{m}=4 ; \mathrm{n}=$ the number of samples on which the FFT is computed, and 
Re denotes the real part of the complex FFT value.

Once the P1 and P2 parameters are computed, these parameters can be used as inputs in the fuzzy decision algorithm. Some fuzzy methods for time series prediction are given in [9], and for nonlinear systems in [10]. In order to further optimize the computation time simple fuzzy rules, easy to evaluate membership functions and inference methods are used. The proposed fuzzy system structure is given below:

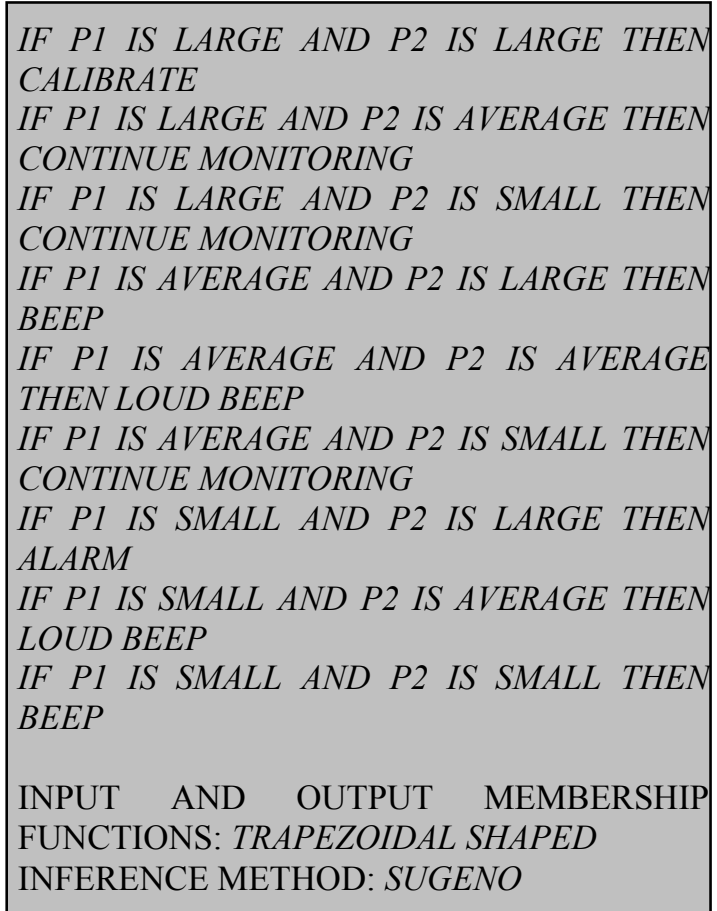

The actual fuzzy system parameters have to be determined in a calibration session of the whole system.

The meanings of decisions which are made are as follows:

- CALIBRATE - in this case there is a decisional conflict between the two parameters (a situation that theoretically could not happen). That is why the system will recommend the fulfillment of the calibration procedure.

- CONTINUE MONITORING - the system continues to execute the algorithm;

- BEEP, LOUD BEEP, ALARM - are three levels of sound intensity of the sound alarm system.

\section{Conclusions}

The proposed drowsiness detection system has to work in real-time conditions so the algorithm for data processing and decision making has to work fast. This is required by the need to alarm the driver or operator in a proper time.

The computation of parameter $\mathrm{P} 1$ is relatively easy and the time required for computation is short enough to allow its use in real-time systems. The second parameter P2 is computed with the FFT algorithm which is also known to be fast especially when it is applied only to 256 samples, which is more than enough for the frequency range needed. Here the main issue is not the processing time (which is about the order of $10^{-4}$ seconds), but the need to detect a minimum of $8 \mathrm{~Hz}$ frequency with an acceptable accuracy. This time is evaluated to be about 0.14 seconds.

The decision time using the presented fuzzy algorithm and considering the selected membership functions and inference method should not take more than a few tenths of milliseconds. This will lead to an overall processing time of about 0.2 up to 0.25 seconds.

\section{REFERENCES}

1. CARLSON, N. R., Sleep and Biological Rhythms. in Physiology of Behavior, 7th ed. Boston: Allyn \& Bacon Publishers, 2001.

2. Sleep Research Society, Basics of Sleep Behavior Syllabus, WebSciences International and Sleep Research Society, 1997.

3. SUlLIVAN, T. J., S. R. DEISS, T.-P. JUNG, G. CAUWENBERGHS, A Brain-Machine Interface using DryContact, Low-Noise EEG Sensors, 2008 IEEE International Symposium on Circuits and Systems, 18-21 May 2008, Washington, US.

4. X. YU, Real-time Non-intrusive Detection of Driver Drowsiness, Intelligent Transportation Systems Institute Center for Transportation Studies, University of Minnesota, May 2009. 
5. DALY, J. J., J. R. WOLPAW, BrainComputer Interfaces in Neurological Rehabilitation, Volume 7, Issue 11, November 2008, pp. 1032-1043.

6. TING, J.-A., A. D'SOUZA, K. YAMAMOTO, T. YOSHIOKA, D. HOFFMAN, S. KAKEIF, L. SERGIO, J. KALASKA, M. KAWATO, P. STRICK, S. SCHAAL, Variational Bayesian Least Squares: An Application to Brain-machine Interface Data, Neural Networks, Volume 21, Issue 8, October 2008, pp. 1112-1131.

7. CVETKOVIC, D., E. D. ÜBEYLI, I. COSIC, Wavelet Transform Feature Extraction from Human PPG, ECG, and EEG Signal Responses to ELF PEMF Exposures: A Pilot Study, Digital Signal Processing, Volume 18, Issue 5, September 2008, pp. 861-874.
8. TING, W., Y. GUO-ZHENG, Y. BANGHUA, S. HONG, EEG Feature Extraction Based on Wavelet Packet Decomposition for Brain Computer Interface, Measurement, Volume 41, Issue 6, July 2008, pp. 618-62.

9. GEORGESCU, V., Fuzzy Time Series Estimation and Prediction: Criticism, Suitable New Methods and Experimental Evidence, Studies in Informatics and Control, Volume 19, Issue 3, 2010.

10. ABID, H., M. CHTOUROU, A. TOUMI, Robust Fuzzy Sliding Mode Controller for Discrete Nonlinear Systems, International Journal of Computers Communications \& Control Volume III No.1, 2008, pp. 6-20 